\title{
MRONJ and implants: the risk of developing necrosis away from surgery
}

Marco Frontera ${ }^{1}$, Pierpaolo De Francesco ${ }^{1}$, paolo vescovi ${ }^{1}$, Maddalena Manfredi $^{1}$, Giulia Ghidini ${ }^{1}$, Marco Meleti $^{1}$ 1 University of Parma

Funding: The author(s) received no specific funding for this work.

Potential competing interests: The author(s) declared that no potential competing interests exist.

\section{Abstract}

This study aimed to evaluate the prevalence of MRONJ in patients treated with prosthetic implant rehabilitation. We evaluated the risk of MRONJ genesis comparing implant surgery with the development of necrosis after correct implant osteointegration. The implants' presence in these patients is a possible risk factor of necrosis development. The presence of implant might cause a reactivation of osteoclasts.

\section{Background}

Medication-related osteonecrosis of the jaws (MRONJ) is a potential pharmacological complication of therapy with bisphosphonates and some monoclonal antibodies as well as biologic agents. This study aimed to evaluate the prevalence of MRONJ in patients treated with prosthetic implant rehabilitation. We evaluated the risk of MRONJ genesis comparing implant surgery with the development of necrosis after correct implant osteointegration. We focused the attention on the implants' presence in these patients like possible risk factor of necrosis development.

\section{Materials and methods}

Our study focused the attention on the analysis of MRONJ sites associated with the presence of implants ${ }^{[1]}$. In particular, we considered two different groups of patients: G1 developed necrosis within ten months of implant surgery; G2 developed necrosis after 10 months of implant placement and osseointegration. We also considered the genesis of MRONJ focused the attention on the localization of necrosis.

\section{Results}

Twenty-eight patients affected by MRONJ are included in the present evaluation. Among these, 21 (75\%) female and 7 (25\%) male, 18 (64.3\%) cancer and 10 (35.7\%) non-cancer with an average age of 63 years old (range 38-80). Considering the patients included in this study, we analyzed thirty-three MRONJ sites. Among these, 21 (63.6\%) associated to cancer and 12 (36.4\%) non cancer, 25 (75.8\%) sites in female and 8 (24.2\%) sites in male.

Our case series describes two groups of implant patients: in G1 7 (21.2\%) MRONJ sites characterized by implant surgery performed less than 10 months before necrosis; in G2 26 (78.8\%) MRONJ sites in which necrosis began 10 months after 
surgery (range 14-222 months). In G2 the presence of osseointegrated implant might be a risk factor for the necrosis development.

Considering the location of the necrosis, we analyzed 17 (51.5\%) sites in lower jaw, 7 (21.2\%) sites in upper jaw and 9 (27.3\%) sites in mandibula e maxilla at the same time. In particular, we analyzed $3(9.1 \%)$ sites localized in anterior sector and $30(90.9 \%)$ in posterior sector.

Based on the AAOMS classification (update 2014), the present study included 15 (45.4\%) sites in stage I, 11 (33.3\%) in stage II and $7(21.2 \%)$ in stage III ${ }^{[2][3]}$.

\section{Conclusions}

Implant surgery is one of the causes of MRONJ more in oncological patients than patients with osteometabolic disorders. Moreover, it's important to consider how the implant placement many years before the beginning of drug therapy is however a risk factor for the MRONJ genesis. In this kind of patients. presence of implant is an important risk factor for MRONJ development, in particular in posterior sectors of mandibula and maxilla. So, presence of implant in patients treated by bisphosphonate or other drugs associated with $\mathrm{ONJ}$ is a risk factor of necrosis development as the implant surgery. The presence of implant might cause a reactivation of osteoclasts ${ }^{[4]}$.

\section{References}

1. ^Ilaria Giovannacci, Marco Meleti, Maddalena Manfredi, Carmen Mortellaro, et al. (2016). Medication-Related Osteonecrosis of the Jaw Around Dental Implants. doi:10.1097/scs.0000000000002564.

2. `Salvatore L. Ruggiero, Thomas B. Dodson, John Fantasia, Reginald Goodday, et al. (2014). American Association of Oral and Maxillofacial Surgeons Position Paper on Medication-Related Osteonecrosis of the Jaw-2014 Update. Journal of Oral and Maxillofacial Surgery, vol. 72 (10), 1938-1956. doi:10.1016/j.joms.2014.04.031.

3. 'Giuseppina Campisi, Rodolfo Mauceri, Francesco Bertoldo, Giordana Bettini, et al. (2020). Medication-Related Osteonecrosis of Jaws (MRONJ) Prevention and Diagnosis: Italian Consensus Update 2020. IJERPH, vol. 17 (16), 5998. doi:10.3390/ijerph17165998.

4. 'Tae-Geon Kwon, Chung-O Lee, Jin-Woo Park, So-Young Choi, et al. (2012). Osteonecrosis associated with dental implants in patients undergoing bisphosphonate treatment. Clin. Oral Impl. Res., vol. 25 (5), 632-640. doi:10.1111/clr.12088. 